\title{
Early evaluation of the apoptotic index ratio is useful in predicting the efficacy of chemoradiotherapy in esophageal squamous cell carcinoma
}

\author{
JUN SOBAJIMA ${ }^{1}$, KENSUKE KUMAMOTO ${ }^{1}$, NORIHIRO HAGA ${ }^{1}$, JUNICHI TAMARU ${ }^{2}$, \\ TAKEO TAKAHASHI ${ }^{3}$, TATSUYA MIYAZAKI ${ }^{4}$ and HIDEYUKI ISHIDA ${ }^{1}$
}

\begin{abstract}
Departments of ${ }^{1}$ Digestive Tract and General Surgery, ${ }^{2}$ Pathology, and ${ }^{3}$ Radiation Oncology, Saitama Medical Center, Saitama Medical University, Saitama; ${ }^{4}$ Department of General Surgical Science, Gunma University Graduate School, Graduate School of Medicine, Gunma, Japan
\end{abstract}

Received July 16, 2011; Accepted October 18, 2011

DOI: $10.3892 / \mathrm{ol} .2011 .468$

\begin{abstract}
Chemoradiotherapy for advanced esophageal cancer is a standard treatment alongside surgical treatment. Although numerous investigators have attempted to identify the predictive markers for chemoradiosensitivity, there appear to be few candidates that can be applied in clinical use. Using biopsy specimens, we investigated the apoptotic index (AI) prior to treatment and following a radiation dose of $10 \mathrm{~Gy}$ to detect the early response to chemoradiotherapy in 28 patients with esophageal squamous cell carcinoma. Molecular markers, including p53, p21, bax, bcl-2, HSP27, HSP70, HSP90, Ku70, Ku86 and HIF-1 $\alpha$, were also examined by immunohistochemical staining. The patients were divided into two groups depending on the response to chemoradiotherapy: a responder group $(\mathrm{RG})(\mathrm{n}=19)$ that included the patients with complete or partial response, and a non-responder group (NRG) $(\mathrm{n}=9)$, that included patients with stable or progressive disease. In the RG and NRG, the AI of pretreatment was $4.7 \pm 5.3$ (mean $\pm \mathrm{SD}$, cells $/ 1,000$ cells) and $5.9 \pm 3.7$, respectively. The apoptotic index ratio (AIR), which was determined by dividing the AI following 10 Gy radiation by the pretreatment $\mathrm{AI}$, was higher in the RG compared to the NRG (4.7 \pm 4.5 versus $1.9 \pm 1.4, p=0.03)$. When the cut-off value of AIR was set at 2.4 , the sensitivity, specificity and accuracy were 74,78 and $76 \%$, respectively. Among the molecular markers we examined immunohistochemically, a positive p53 expression in the pretreatment evaluation was associated with the efficacy of chemoradiotherapy $(\mathrm{p}=0.08)$. Regarding the expression of other molecular markers, no significant correlations were found in RG and NRG. In the present study, the results indicated that
\end{abstract}

Correspondence to: Dr Hideyuki Ishida, Department of Digestive Tract and General Surgery, Saitama Medical Center, Saitama Medical University, 1981 Kamoda, Kawagoe, Saitama 350-8550, Japan

E-mail:05hishi@saitama-med.ac.jp

Key words: apoptotic index, chemoradiotherapy, esophageal squamous cell carcinoma
AIR is useful for the prediction of chemoradiosensitivity in esophageal squamous cell carcinoma.

\section{Introduction}

Findings of the majority of meta-analyses of randomized trials with surgery versus surgery and chemoradiotherapy for advanced esophageal cancer showed that preoperative chemoradiotherapy is beneficial in improving outcome for patients with locally advanced esophageal cancer compared with surgery alone (1-4). Despite recent advances in multimodality treatment, complete pathological response to chemoradiotherapy occurred in $10-45.5 \%$ of patients with advanced esophageal cancer (5). Patients with advanced esophageal cancer are not likely to reap any benefits from chemoradiotherapy. The prediction of chemoradiosensitivity for patients with advanced esophageal cancer is necessary to determine the therapeutic course prior to treatment. Various studies have identified candidates for molecular markers to predict the efficacy of chemoradiotherapy for advanced esophageal cancer using biopsy specimens prior to chemoradiotherapy (6-14). These studies demonstrated the correlation between the response of chemoradiotherapy and the expression of molecular markers including apoptosis, cell cycle, stress, DNA damage and proliferation-related genes. Among them, p53 and its related genes, p21 and bax, which play a pivotal role in the regulation of cell fate, have been investigated the most in the evaluation of chemoradiosensitivity (7-10). Regarding apoptosis-related genes, bcl-2 is also known to act as an apoptotic suppressor (10). Moreover, the heat shock protein family, including HSP27, HSP70 and HSP90, has been demonstrated to have anti-apoptotic effects and is resistant to chemo- and radiotherapy (11). In particular, apoptosis induced by radiation occurred due to DNA double-strand breaks that are predominantly repaired by the binding of Ku70 and Ku86 proteins to the free DNA ends followed by recruitment of the catalytic subunit of DNA-dependent protein kinase $(12,13)$. Hypoxic conditions with hypoxia-inducible factor $1 \alpha$ (HIF-1 $\alpha)$ expression also are resistant to chemotherapy and radiotherapy (14). Although these molecules may contribute to predicting the efficacy of chemoradiotherapy in esophageal 
cancer, at present there is no consensus regarding the application of these markers in clinical practice.

In this study, we focused on the detection of early response to chemoradiotherapy to determine the appropriate therapeutic course, resulting in the prevention of severe adverse events, and reduction of the costs involved for patients with esophageal cancer. Thus, we investigated the apoptotic index (AI) immediately prior to treatment and following a radiation dose of 10 Gy using biopsy specimens. Additionally, we examined molecular marker candidates for chemoradiosensitivity, including p53, p21, bax, bcl-2, HSP27, HSP70, HSP90, $\mathrm{Ku} 70, \mathrm{Ku} 86$ and HIF-1 $\alpha$, as previously reported (6-14).

\section{Materials and methods}

Patients and tissue samples. Biopsy specimens were obtained endoscopically from 28 patients (26 males and 2 females) with esophageal squamous cell carcinoma prior to chemoradiotherapy or radiotherapy and following a radiation dose of 10 Gy in one week at the Saitama Medical Center, Japan, between November 2007 and December 2008. The median age of the patients was 70 years (range 52-83). The location of the primary tumor was as follows: cervical esophagus in 1 patient, upper thoracic esophagus in 8 patients, middle thoracic esophagus in 12 patients and lower thoracic esophagus in 7 patients. Tumor stage was classified according to the sixth edition of the TNM classification of the International Union against Cancer (UICC) (15). Tumor stage: stage I, 4 patients; stage II, 5 patients; stage III, 14 patients; and stage IV, 5 patients. Biopsy specimens were fixed in formalin, embedded in paraffin and sectioned at a thickness of $4 \mu \mathrm{m}$. The samples were examined following hematoxylin and eosin staining. Immunohistochemical studies and an apoptosis study were also performed. Written informed consent to participate in the study was obtained from each patient prior to treatment, according to the ethics guidelines of Saitama Medical Center, Saitama Medical University.

Treatment protocol. Following diagnosis, the patients underwent radiotherapy or chemoradiotherapy, consisting of concurrent radiotherapy and chemotherapy, for 7 weeks. External-beam radiotherapy was delivered by a two-field technique using a $10 \mathrm{MV}$ photon beam and the total dose was 60-70 Gy in 30-35 fractions. Radiation therapy without concurrent chemotherapy was performed in 4 patients. A total of 24 patients received the following concurrent chemotherapy: 13 patients underwent a regimen of $70 \mathrm{mg} / \mathrm{m}^{2}$ cisplatinum administered intravenously over $1 \mathrm{~h}$ on days 1 and 29, and $700 \mathrm{mg} / \mathrm{m}^{2}$ 5-fluorouracil (5-FU) administered as a continuous intravenous infusion on days 1-5 and 29-33. Two patients received $6.6 \mathrm{mg} / \mathrm{m}^{2}$ cisplatinum administered intravenously over $1 \mathrm{~h}$ on days 1-5, 8-12 and 29-33, and $350 \mathrm{mg} / \mathrm{m}^{2} 5-\mathrm{FU}$ was administered as a continuous intravenous infusion on days 1-5, 8-12 and 29-33. Nine patients received the regimen that consisted of $6.6 \mathrm{mg} / \mathrm{m}^{2}$ docetaxel administered intravenously over $1.5 \mathrm{~h}$ on days $1,8,15,22,29$ and 37.

Clinical features and evaluation of treatment. Prior to treatment, all 28 patients were evaluated by radiographic examination (chest X-rays and barium swallow), endoscopy (esophagoscopy and in certain cases bronchoscopy), endoscopic ultrasonography and computed tomography (CT). Biopsy samples were obtained from three or more points. The clinical response was evaluated 2 weeks following the end of treatment according to the Response Evaluation Criteria in Solid Tumors (RECIST) (16) and the guidelines of the Japanese Society for Esophageal Diseases (JSED) $(17,18)$. When the case had a lesion that could be evaluated, we evaluated the response according to RECIST. When such a lesion was not present, we evaluated the response according to the guidelines of JSED. Assessment included repeated endoscopy and CT scans. Endoscopy was carried out by two investigators. All 28 patients underwent a CT scan of the neck, chest and abdomen. CT was performed at a slice width of $10 \mathrm{~mm}$ from the neck to the bottom of the liver using an intravenous contrast medium. The response was classified as complete response, partial response $(>30 \%$ decrease), stable disease $(<30 \%$ decrease) and progressive disease in the treatment lesion.

Immunohistochemical staining. Immunohistochemical staining was performed by the labeled streptavidin biotinylated antibody (LSAB) method using a Super Sensitive Detection Kit (BioGenex Laboratories, Inc., CA, USA). Paraffin-embedded histological sections $(4-\mu \mathrm{m})$ were deparaffinized and washed with water, and then endogenous peroxidase activity was blocked with $0.3 \% \mathrm{H}_{2} \mathrm{O}_{2}$ in methanol. After washing in phosphatebuffered saline (PBS), a blocking agent (Dako, Glostrup, Denmark) was used to regulate non-specific reactivity. For antigen retrieval, the sections were autoclaved at $121^{\circ} \mathrm{C}$ for $15 \mathrm{~min}$ or in a microwave oven for $10 \mathrm{~min}$. These sections were then incubated with the primary antibodies at room temperature for $60 \mathrm{~min}$ or overnight. After washing in PBS, tissues were incubated with biotin-labeled anti-rabbit or antimouse secondary antibodies for $20 \mathrm{~min}$ at room temperature and then reacted with streptavidin-biotin horseradish peroxidase complex for $20 \mathrm{~min}$. Immunostaining was visualized by develo-ping the slides in diaminobenzidine (DAB). The sections were lightly counterstained with hematoxylin. Negative controls were prepared by substituting blocking buffer for each primary antibody, and no detectable staining was evident.

Antibodies were purchased from the following manufacturers: monoclonal antibody (Mab) specific for p53 (DO-7; DAKO A/S, Glostrup, Denmark; 1:40), Mab specific for $\mathrm{p} 21$ (4D10; Novocastra Laboratories Ltd., Newcastle, UK; 1:20), Mab specific for bax (sc-7480; Santa Cruz Biotechnology, Inc., Santa Cruz, CA, USA; 1:100), Mab specific for bcl-2 (124; DAKO A/S; 1:100), Mab specific for HSP27 (G3.1; StressGen Biotechnologies Corporation, Victoria, BC, Canada; 1:400), Mab specific for HSP70 (C92F3A-5; StressGen Biotechnologies Corporation; 1:100), Mab specific for HSP90 (k3705; StressGen Biotechnologies Corporation; 1:600), Mab specific for Ku70 (M-19; Santa Cruz Biotechnology, Inc.; 1:500), Mab specific for Ku86 (M-20; Santa Cruz Biotechnology, Inc.; 1:20) and Mab specific for HIF-1 $\alpha$ (OZ12; Thermo Fisher Scientific Inc. Waltham, MA, USA; $1: 10)$.

TUNEL method. Apoptotic cells were determined by the TUNEL method. Apoptotic cells were visualized using the 
Table I. Correlation between clinicopathological characteristics and response to chemoradiotherapy.

\begin{tabular}{|c|c|c|c|}
\hline & $\begin{array}{c}\text { Responders } \\
(\mathrm{n}=19)\end{array}$ & $\begin{array}{l}\text { Non-responders } \\
\qquad(\mathrm{n}=9)\end{array}$ & P-value \\
\hline Age median (range) & $74(62-80)$ & $68(52-83)$ & 0.27 \\
\hline Gender & & & 0.99 \\
\hline Male $(n=26)$ & 18 & 8 & \\
\hline Female $(\mathrm{n}=2)$ & 1 & 1 & \\
\hline Location & & & 0.27 \\
\hline Cervical $(\mathrm{n}=1)$ & 1 & 0 & \\
\hline Upper thoracic $(\mathrm{n}=8)$ & 7 & 1 & \\
\hline Middle thoracic $(\mathrm{n}=12)$ & 8 & 4 & \\
\hline Lower thoracic $(\mathrm{n}=7)$ & 3 & 4 & \\
\hline Depth of invasion & & & 0.14 \\
\hline $\mathrm{T} 1(\mathrm{n}=4)$ & 4 & 0 & \\
\hline $\mathrm{T} 2(\mathrm{n}=5)$ & 3 & 2 & \\
\hline T3 $(n=15)$ & 8 & 7 & \\
\hline $\mathrm{T} 4(\mathrm{n}=4)$ & 4 & 0 & \\
\hline Regional lymph node & & & 0.44 \\
\hline N0 $(n=9)$ & 7 & 2 & \\
\hline $\mathrm{N} 1(\mathrm{n}=19)$ & 12 & 7 & \\
\hline Distant metastasis & & & 0.68 \\
\hline $\mathrm{M} 0(\mathrm{n}=23)$ & 16 & 7 & \\
\hline $\mathrm{M} 1(\mathrm{n}=5)$ & 3 & 2 & \\
\hline Stage & & & 0.52 \\
\hline $\mathrm{I}(\mathrm{n}=4)$ & 4 & 0 & \\
\hline II $(n=5)$ & 3 & 2 & \\
\hline III $(n=14)$ & 9 & 5 & \\
\hline IV $(n=5)$ & 3 & 2 & \\
\hline
\end{tabular}

ApopTag Plus peroxidase in situ apoptosis detection kit (Chemicon International, Temecula, CA, USA). Formalin-fixed, paraffin-embedded sections were deparaffinized in xylene and dehydrated through graded alcohol. Briefly, following routine deparaffinization, sections were incubated with proteinase $\mathrm{K}$ for $15 \mathrm{~min}$ at room temperature. The sections were washed twice in distilled water for 2 min each time and incubated with $3 \% \mathrm{H}_{2} \mathrm{O}_{2}$ for $5 \mathrm{~min}$. The sections were subjected to enzymic homopolymeric tailing with $\mathrm{TdT}$ and digoxigenin-labeled nucleotides for $60 \mathrm{~min}$ at room temperature in a humidified atmosphere. The nucleotides incorporated were revealed by incubation with anti-digoxigenine antibody bound to peroxidase for $30 \mathrm{~min}$ at room temperature. Immunostaining was visualized by developing the slides in DAB. Counterstaining was performed with methyl green.

Evaluation of molecular marker expression and the AI. Expression of molecular markers was evaluated as positive when the nucleus and/or cytoplasm of cancer tissue indicated at least $5 \%$ dye-affinity when the total field of view was observed at a magnification of $\mathrm{x} 40$. The AI was statistically averaged as the number of positive cells among $>1,000$ tumor cells counted in three randomly selected areas. The AI ratio
(AIR) was determined by dividing the AI following 10 Gy radiation by the pretreatment AI. Assessment of the staining was evaluated by two independent pathologists who had no access to data pertaining to the clinical status of the patients.

Statistical analysis. Associations between categorical variables and the expression of molecular markers were evaluated with the Fisher's exact probability test, Mann-Whitney's U test, Wilcoxon signed-ranks test and the paired t-test. A receiver operating characteristics (ROC) curve was generated to determine the optimal cut-off value for diagnostic discrimination. The differences at P-values $<0.05$ were considered significant, and all P-values reported were two-sided. Statistical analyses were carried out using Statflex (ver6.0 Artec, Osaka, Japan).

\section{Results}

Correlation between clinicopathological characteristics and the response to chemoradiotherapy. Regarding the efficacy of chemoradiotherapy, there was a complete response in 5 patients $(17.9 \%)$, a partial response in 14 patients $(50 \%)$, stable disease in 6 patients $(21.4 \%)$ and progressive disease in 3 patients $(10.7 \%)$. The patients were divided into two groups 

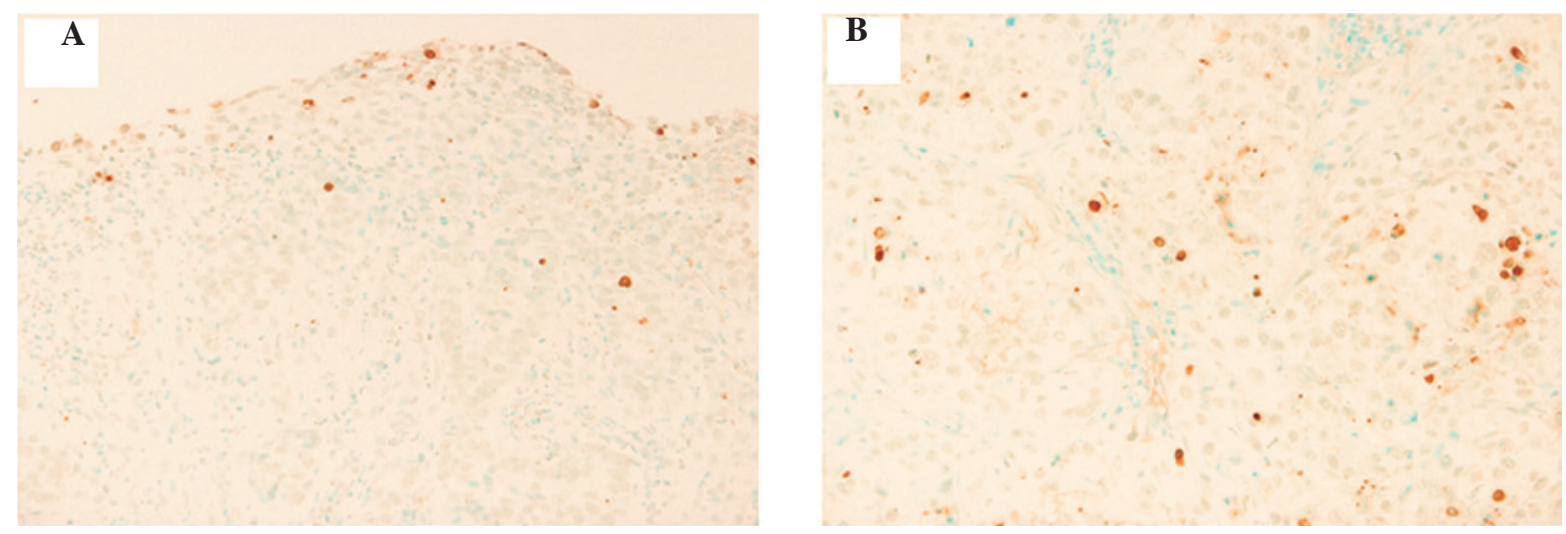

Figure 1. The representative pattern of apoptotic cells by a TUNEL method in esophageal squamous cell carcinoma. (A) Apoptotic cells were detected by a TUNEL method using a biopsy specimen prior to treatment in the representative case (magnification, $\mathrm{x} 40$ ). (B) Apoptotic cells were detected by a TUNEL method using the biopsy specimen following a radiation dose of $10 \mathrm{~Gy}$ in the same case (magnification, $\mathrm{x} 40$ ).

Table II. Apoptotic index.

\begin{tabular}{lccc}
\hline & $\begin{array}{c}\text { Responders } \\
(\mathrm{n}=19)\end{array}$ & $\begin{array}{c}\text { Non-responders } \\
(\mathrm{n}=9)\end{array}$ & P-value \\
\hline Pretreatment & $4.7 \pm 5.3$ & $5.9 \pm 3.7$ & 0.18 \\
After 10 Gy & $12.9 \pm 6.8$ & $11.0 \pm 10$ & 0.36 \\
\hline
\end{tabular}

Mann-Whitney U test.

depending on the response: responder group (RG) $(n=19)$, including the patients with complete or partial response, and the non-responder group (NRG) (n=9), which included patients with stable or progressive disease. The correlation between the patient characteristics and the response to chemoradiotherapy is shown in Table I. No correlations were statistically observed between RG and NRG in terms of age, gender, tumor location, depth of invasion, regional lymph node, distant metastasis and UICC stage.

$A I$ comparing with $R G$ and $N R G$. Using biopsy specimens, the AI was examined prior to treatment and following a radiation dose of $10 \mathrm{~Gy}$ in RG and NRG (Fig. 1A and B). In RG and NRG, AI of pretreatment was 4.7 \pm 5.3 (cells/1000 cells) and $5.9 \pm 3.7$, respectively $(\mathrm{P}=0.18)$. Following a radiation dose of $10 \mathrm{~Gy}$, the AI was $12.9 \pm 6.8$ in RG and $11.0 \pm 10$ in NRG $(\mathrm{P}=0.36)$. No statistically significant difference was found between these groups prior to treatment and following $10 \mathrm{~Gy}$ radiation (Table II).

We then analyzed the AIR of prior to treatment and $10 \mathrm{~Gy}$ radiation. AIR was found to be $4.7 \pm 4.5$ in RG and $1.9 \pm 1.4$ in NRG. The AIR of RG was significantly higher than that of NRG ( $\mathrm{P}=0.03$ ) (Fig. 2). When the ROC curve was generated to determine the optimal cut-off value for predicting the response of chemoradiotherapy using AIR, the area under the ROC curve was 0.78 (Fig. 3). Based on the result of the ROC curve, when the cut-off value of AIR was set at 2.4, the sensitivity, specificity and accuracy were 74,78 and $76 \%$, respectively.

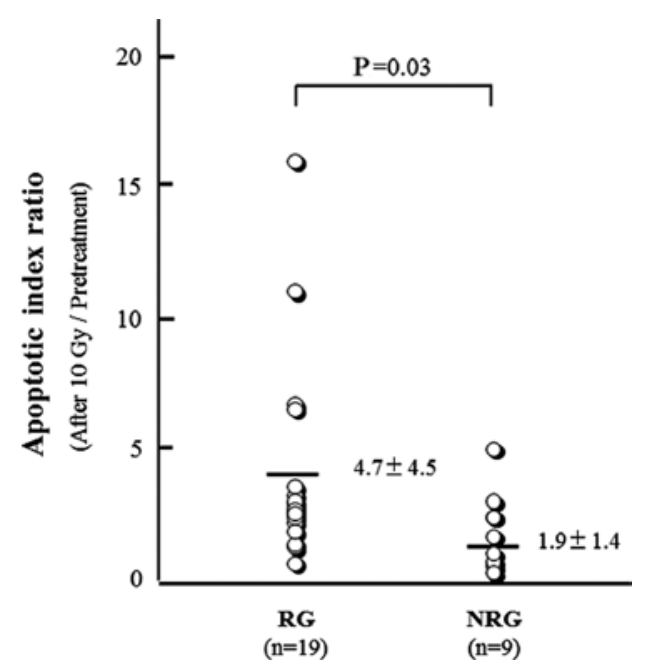

Figure 2. Comparison of the apoptotic index ratio (AIR) between responder (RG) and non-responder groups (NRG). AIR was calculated to compare the AI prior to treatment with that after a radiation dose of $10 \mathrm{~Gy}$. The AIR of RG was significantly higher than that of NRG. Statistical analysis was performed by a Mann-Whitney U test. Each mean \pm SD of RG and NRG is shown.

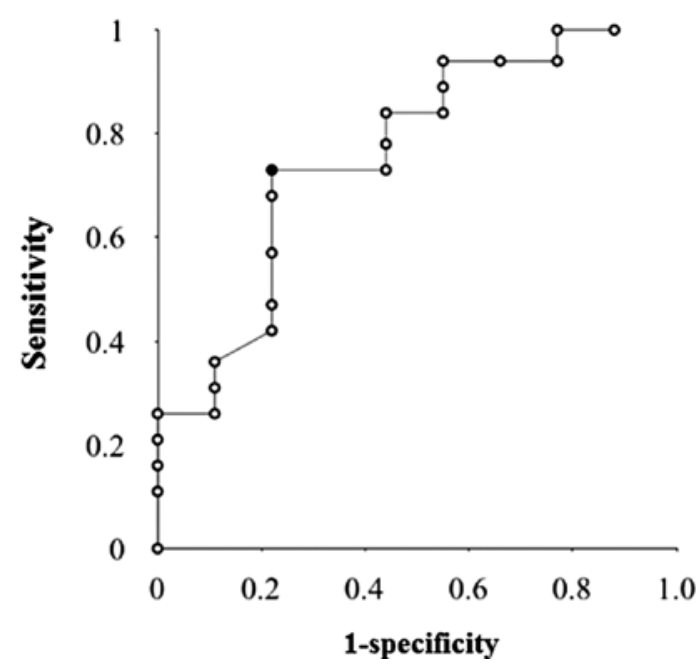

Figure 3. The ROC curve for the optimal cut-off value for predicting the response of chemoradiotherapy using apoptotic index ratio (AIR). Based on the result of ROC curve, when a cut-off value of AIR was set at 2.4, the sensitivity, specificity and accuracy were 74,78 and $76 \%$, respectively. 
Correlation between the expression of molecular markers and the chemoradiosensitivity. Using biopsy specimens, we examined the expression of the molecular markers, including p53, p21, bax, bcl-2, HSP27, HSP70, HSP90, Ku70, Ku86 and HIF-1 $\alpha$, immunohistochemically prior to treatment and following a radiation dose of $10 \mathrm{~Gy}$, and analyzed the correlation between the protein expression and chemoradiosensitivity. The positive expression of p53 prior to treatment is associated with the efficacy of chemoradiotherapy, no statistical significance was observed $(\mathrm{p}=0.08)$. Regarding further molecular expression in biopsy specimens obtained prior to treatment and following a radiation dose of $10 \mathrm{~Gy}$, no significant correlations were found in RG and NRG.

\section{Discussion}

The candidates for molecular markers for the prediction of chemoradiosensitivity for esophageal cancer have been investigated to identify the responders or non-responders prior to treatment. Chemoradiosensitivity may be used to allow appropriate treatment to be administered for predicted responders and to prevent severe adverse events and save costs for predicted non-responders. A number of molecular markers have been examined retrospectively and reported as good predictors for chemoradiotherapy in esophageal cancer (6-14). It is reasonable that most of these markers are associated with the apoptotic pathway. However, consensus regarding the application of these markers in clinical practice has not been reached. The aim of the present study was to find the appropriate marker for the prediction of tumor response against chemoradiosensitivity at a relatively early step, and then focus on the observation of the apoptotic cells directory using a TUNEL method as an AI. Based on this hypothesis, we compared the AI of biopsy specimens following a radiation dose of $10 \mathrm{~Gy}$ with that prior to treatment. As a result, we found that AIR reflected the efficacy of chemoradiotherapy. AIR of RG was significantly higher than that of NRG. Furthermore, the sensitivity, specificity and accuracy were approximately $75 \%$ when the cut-off value of AIR was set at 2.4. These results appear to be significant.

We chose the evaluation point of 10 Gy (5 days) following radiation. The clinically crucial objective is to make a go/no-go decision for chemoradiotherapy by precise assessment of chemoradiosensitivity at a relatively early stage. Although we are not able to emphasize the reason for 10 Gy being the most appropriate dose to judge the early efficacy of chemoradiotherapy, the evaluation of the apoptotic state at $10 \mathrm{~Gy}$ appears to be an acceptable dose to satisfy the clinical value as mentioned above. A radiation dose of 9 Gy reportedly induces recruitment of cancer cells from the heterogeneous cell cycling population to the synchronous cycling cell population, resulting in the enhancement of radiosensitivity (19). As a result, the high growth fraction at 9 Gy was associated with a favorable prognosis in cervical cancer patients (19). Moreover, another study demonstrated that evaluation following $10 \mathrm{~Gy}$ doses of radiotherapy was useful in predicting the response to radiotherapy in oral squamous cell carcinoma (20). In in vitro experiments, 2-10 Gy radiation substantially induced apoptosis using cancer cells $(21,22)$. We observed an apparent increase of apoptotic cells in numerous biopsy specimens obtained following a radiation dose of 10 Gy compared with that prior to treatment. Therefore, our results suggest that the assessment of AIR was appropriate to predict the efficacy of chemoradiotherapy for the patients with advanced esophageal squamous cell carcinoma.

We further examined the expression of molecular markers, including p53, p21, bax, bcl-2, HSP27, HSP70, HSP90, $\mathrm{Ku} 70$, Ku86 and HIF-1 $\alpha$, which were previously shown to be candidates for the prediction of chemoradiosensitivity. In our results, the expression of these markers was not associated with the efficacy of chemoradiotherapy using biopsy specimens obtained prior to treatment. Moreover, the marked alteration of these markers was not observed in tissues following a radiation dose of 10 Gy when compared with that prior to treatment. Among those molecules, p53-positive expression in pretreatment tissues had a tendency to link to the efficacy of chemoradiotherapy. The p53 gene is one of the most investigated genes and is mutated in approximately $50 \%$ of cases in numerous types of cancer. Intensive studies have suggested that wild-type p53 acts as a key inducer for apoptosis and cell cycle arrest against anti-cancer agents and radiation, while mutated p53 has achieved radioresistance and chemoresistance. However, p53 status does not necessarily correspond to the clinical value. A number of reports have shown that the patients with immunohistochemically p53-positive expression have a good prognosis and chemosensitivity in esophageal cancer $(7,10)$, although the opposite results have also been shown $(8,23,24)$. Another argument is that p53 status is not associated with chemoradiosensitivity and prognosis for patients with esophageal cancer $(25,26)$. Based on these results, even p53 may be unsuitable as a predictive marker of chemoradiosensitivity. Therefore, it is unlikely that a single molecular marker to predict the efficacy of chemoradiotherapy can be identified.

In the case that patients with early stages of esophageal cancer have high-risk factors for surgical treatment or desire to preserve laryngeal function, chemoradiotherapy would be administered, although surgical treatment is also likely to produce favorable results. If we were able to predict at an earlier stage that the response for chemoradiotherapy is likely to be poor, then surgical treatment should be re-considered for patients with early stage cancer during chemoradiotherapy. That is certainly the case with patients with advanced stage. Therefore, our study appears to provide significant results, despite certain limitations. For example, the number of clinical cases was small and the regimens of chemotherapy combined with radiotherapy were not integrated. Based on the results, the cases treated with identical regimens of chemoradiotherapy for esophageal cancer are to be utilized in the future to verify our current results.

In the present study, we found that the comparison of AI between biopsy tissues obtained prior to treatment and following a radiation dose of $10 \mathrm{~Gy}$ was clinically significant for the prediction of chemoradiosensitivity in esophageal squamous cell carcinoma. Consequently, our findings may contribute to determining the therapeutic course, resulting in the prevention of severe adverse events and reduced costs for the predicted non-responders with esophageal squamous cell carcinoma. 


\section{References}

1. Greer SE, Goodney PP, Sutton JE and Birkmeyer JD: Neoadjuvant chemoradiotherapy for esophageal carcinoma: A meta-analysis. Surgery 137: 172-177, 2005.

2. Fiorica F, Di Bona D, Schepis F, Licata A, Shahied L, Venturi A, Falchi AM, Craxì A and Cammà C: Preoperative chemoradiotherapy for oesophageal cancer: A systematic review and meta-analysis. Gut 53: 925-930, 2004.

3. Urschel JD and Vasan H: A meta-analysis of randomized controlled trials that compared neoadjuvant chemoradiation and surgery to surgery alone for resectable esophageal cancer. Am J Surg 185: 538-543, 2003

4. Tepper J, Krasna MJ, Niedzwiecki D, Hollis D, Reed CE, Goldberg R, Kiel K, Willett C, Sugarbaker D and Mayer R: Phase III trial of trimodality therapy with cisplatin, fluorouracil, radiotherapy, and surgery compared with surgery alone for esophageal cancer: CALGB 9781. J Clin Oncol 26: 1086-1092, 2008.

5. Lv J, Cao XF, Zhu B, Ji L, Tao L and Wang DD: Effect of neoadjuvant chemoradiotherapy on prognosis and surgery for esophageal carcinoma. World J Gastroenterol 15: 4962-4968, 2009.

6. Theisen J, Krause B, Peschel C, Schmid R, Geinitz H, and Friess $\mathrm{H}$ : Early response evaluation and prediction in neoadjuvant-treated patients with esophageal cancer. World J Gastrointest Surg 1: 30-37, 2009.

7. Muro K, Ohtsu A, Boku N, Chin K, Oda Y, Fujii T, Hosokawa K, Yoshida S and Hasebe T: Association of p53 protein expression with responses and survival of patients with locally advanced esophageal carcinoma treated with chemoradiotherapy. Jpn J Clin Oncol 26: 65-69, 1996.

8. Shimoyama S, Konishi T, Kawahara M, Aoki F, Harada N, Shimizu S, Murakami $\mathrm{T}$ and Kaminishi M: Expression and alteration of $\mathrm{p} 53$ and $\mathrm{p} 21$ (waf1/cip1) influence the sensitivity of chemoradiation therapy for esophageal cancer. Hepatogastroenterology 45: 1497-1504, 1998.

9. Kitamura K, Saeki H, Kawaguchi H, Araki K, Ohno S, Kuwano H, Maehara Y and Sugimachi K: Immunohistochemical status of the p53 protein and $\mathrm{Ki}-67$ antigen using biopsied specimens can predict a sensitivity to neoadjuvant therapy in patients with esophageal cancer. Hepatogastroenterology 47: 419-423, 2000

10. Shimoji H, Miyazato H, Nakachi A, Kuniyoshi S, Isa $T$, Shiraishi M, Muto Y and Toda T: Expression of p53, bcl-2, and bax as predictors of response to radiotherapy in esophageal cancer. Dis Esophagus 13: 185-190, 2000.

11. Jego G, Hazoumé A, Seigneuric R, and Garrido C: Targeting heat shock proteins in cancer. Cancer Lett: 13 November, 2010 (E-pub ahead of print).

12. Gullo C, Au M, Feng G and Teoh G: The biology of Ku and its potential oncogenic role in cancer. Biochim Biophys Acta 1765: 223-234, 2006.

13. Hefferin ML and Tomkinson AE: Mechanism of DNA double-strand break repair by non-homologous end joining. DNA Repair (Amst) 4: 639-648, 2005.
14. Sohda M, Ishikawa $H$, Masuda $N$, Kato $H$, Miyazaki $T$, Nakajima M, Fukuchi M, Manda R, Fukai Y, Sakurai H and Kuwano H: Pretreatment evaluation of combined HIF-1alpha, p53 and p21 expression is a useful and sensitive indicator of response to radiation and chemotherapy in esophageal cancer. Int J Cancer 110: 838-844, 2004.

15. Sobin LH and Wittekind C (eds): TNM Classification of Malignant Tumours: Sixth edition. Wiley-Liss, New York, NY, pp72-76, 2002.

16. Therasse P, Arbuck SG, Eisenhauer EA, Wanders J, Kaplan RS, Rubinstein L, Verweij J, Van Glabbeke M, van Oosterom AT, Christian MC and Gwyther SG: New guidelines to evaluate the response to treatment in solid tumors. J Natl Cancer Inst 92: 205-216, 2000.

17. Japanese Society for Esophageal Diseases. Guidelines for the clinical and pathologic studies on carcinoma of the esophagus, ninth edition: Preface, general principles, part I. Esophagus 1; 61-88, 2004.

18. Japan Esophageal Society. Japanese Classification of Esophageal Cancer. 10th ed., Kanehara, Tokyo, 2007.

19. Oka K, Suzuki Y and Nakano T: High growth fraction at 9 grays of radiotherapy is associated with a good prognosis for patients with cervical squamous cell carcinoma. Cancer 89: 1526-1531, 2000.

20. Valente G, Orecchia R, Gandolfo S, Arnaudo M, Ragona R, Kerim S and Palestro G: Can Ki67 immunostaining predict response to radiotherapy in oral squamous cell carcinoma? J Clin Pathol 47: 109-112, 1994.

21. Raju U, Ariga H, Koto M, Lu X, Pickett J, Valdecanas D, Mason KA and Milas L: Improvement of esophageal adenocarcinoma cell and xenograft responses to radiation by targeting cyclin-dependent kinases. Radiother Oncol 80: 185-191, 2006.

22. Mitsuhashi N, Takahashi T, Sakurai H, Nozaki M, Akimoto T, Hasegawa M, Saito Y, Matsumoto H, Higuchi K, Maebayashi K and Niibe $\mathrm{H}$ : A radioresistant variant cell line, NMT-1R, isolated from a radiosensitive rat yolk sac tumour cell line, NMT-1: differences of early radiation-induced morphological changes, especially apoptosis. Int J Radiat Biol 69: 329-336, 1996.

23. Miyata H, Doki Y, Shiozaki H, Inoue M, Yano M, Fujiwara Y, Yamamoto H, Nishioka K, Kishi K and Monden M: CDC25B and p53 are independently implicated in radiation sensitivity for human esophageal cancers. Clin Cancer Res 6: 4859-4865, 2000.

24. Matsubara H, Kimura M, Sugaya M, Koide Y and Gunji Y, Takegana K, Asano T, Ochiai T, Isono K, Sakiyama S and Tagawa M: Expression of wild-type p53 gene confers increased sensitivity to radiation and chemotherapeutic agents in human esophageal carcinoma cells. Int J Oncol 14: 1081-1085, 1999.

25. Ito T, Kaneko K, Makino R, Ito H, Konishi K, Kurahashi T, Kitahara T and Mitamura K: Prognostic value of p53 mutations in patients with locally advanced esophageal carcinoma treated with definitive chemoradiotherapy. J Gastroenterol 36: 303-311, 2001.

26. Sarbia M, Porschen R, Borchard F, Horstmann O, Willers R and Gabbert HE: p53 protein expression and prognosis in squamous cell carcinoma of the esophagus. Cancer 74: 2218-2223, 1994. 